Cahiers d'études africaines

198-199-200|2010

50 ans

\title{
Espace et sacralité
}

\section{Marc Augé}

\section{(2) OpenEdition}

Journals

Édition électronique

URL : https://journals.openedition.org/etudesafricaines/16406

DOI : 10.4000/etudesafricaines. 16406

ISSN : 1777-5353

\section{Éditeur}

Éditions de l'EHESS

\section{Édition imprimée}

Date de publication : 20 novembre 2010

Pagination : 853-856

ISBN : 978-2-7132-2252-8

ISSN : 0008-0055

\section{Référence électronique}

Marc Augé, «Espace et sacralité », Cahiers d'études africaines [En ligne], 198-199-200 | 2010, mis en ligne le 02 janvier 2013, consulté le 21 avril 2022. URL : http://journals.openedition.org/ etudesafricaines/16406; DOI : https://doi.org/10.4000/etudesafricaines.16406

Ce document a été généré automatiquement le 21 avril 2022

(c) Cahiers d'Études africaines 


\section{Espace et sacralité}

\section{Marc Augé}

1 L'espace sacré, en Europe, s'inscrit dans un paysage. Il y a par exemple un paysage catholique classique, familier, que l'on retrouve dans des pays comme l'Espagne, la France et l'Italie: le clocher qui émerge des toits du village, rassemblés autour de l'église comme le troupeau autour du berger. Anciennement, et il existe encore des traces de cette organisation, les tombes des morts entouraient l'église comme pour rendre encore plus visible la communauté des chrétiens. On peut faire trois remarques à propos de cette disposition spatiale :

2 - Elle est indissociable d'une organisation corrélative du temps. L'année, la semaine, les heures du jour sont marquées par des temps forts. Jacques Le Goff a souligné l'importance qu'avait eue de ce point de vue l'introduction au Moyen-Âge des sonneries des cloches.

3 - Elle est planétaire, les églises étant orientées en principe vers l'Orient et Jérusalem. Rome est la capitale du catholicisme et c'est de Rome, comme en témoignent de nombreuses images, que l'Église et le pape s'adressent au monde.

4 - Elle est aussi intérieure. D'une part, traditionnellement (car l'Église a su évoluer), l'espace intérieur de l'église assigne des places spécifiques aux célébrants et aux participants et, parmi ces derniers, à chacun des deux sexes; d'autre part, l'autel et le tabernacle, qui focalisent l'attention et les regards dans l'horizontalité, et l'architecture aérienne de la nef et du chœur, qui propulse ces mêmes regards dans la verticalité, soutiennent, physiquement et métaphoriquement à la fois, l'effort individuel d'élévation de l'âme.

5 Mon expérience d'ethnologue m'a confronté à des types d'organisation spatiale différents, correspondant à des expériences religieuses elles aussi différentes. J'en évoquerai deux. En Afrique, j'ai d'abord fait l'expérience du prophétisme ivoirien, réaction locale à la colonisation et à la prédication chrétienne, qui s'est traduite notamment par des innovations spatiales significatives. Au Togo, j'ai observé directement le fonctionnement de cultes de type vodun dont l'équivalent ivoirien avait plus ou moins disparu dès la première moitié $\mathrm{du} \mathrm{xx}^{\mathrm{e}}$ siècle sous l'action du prophétisme. 
6 Significativement, les prophètes ivoiriens essayaient d'abord d'exprimer spatialement le changement dont ils se voulaient les témoins et les artisans. Je résume d'un mot le prophétisme africain : partout en Afrique sont apparus au moment de la colonisation des «prophètes» qui essayaient d'adapter l'enseignement chrétien à l'Afrique et réciproquement. Certains d'entre eux ont même prétendu réincarner le Christ revenu sur terre pour les Noirs. Tous ces prophètes (ou prophétesses, car il y eut quelques femmes dans ce mouvement) étaient assez proches des conceptions traditionnelles africaines et leur rôle de guérisseur ou de chasseur de sorciers leur conférait une certaine ambivalence. Mais tous avaient conscience de devoir parler au nom de tous et, pour ce faire, de devoir échapper à la dimension locale des guérisseurs traditionnels. Ils ont donc en général créé leur propre lieu original, fondateur, parfois appelé Jérusalem, pour y accueillir des fidèles, des malades ou des accusés de sorcellerie originaires d'autres régions et d'autres ethnies que les leurs. Certains ont mieux réussi que d'autres, mais tous ont emprunté le même chemin, qui a même conduit certains d'entre eux à édifier des cathédrales imposantes.

7 Il y a eu dans ces tentatives un caractère mimétique évident, mais aussi une conscience claire des enjeux du changement de paradigme religieux. La plupart des prophètes ont tenté sans succès de se faire reconnaître par l'une des églises chrétiennes ou par l'un des hôpitaux du pays pour échapper au rôle et à l'image du guérisseur local. Ce que les prophètes ont compris, sans réussir à en tirer toutes les conséquences, c'est que, dans le monde nouveau, le monde né de la colonisation, l'espace, y compris l'espace religieux, se recomposait. Pour leur part, ils ont essayé de reconstituer quelque chose de la double centralité exprimée par le christianisme : centralité dans l'organisation du culte lui-même (l'autel), dans l'organisation du paysage (l'église) et centralité planétaire (allusion à la Nouvelle Jérusalem, résurrection d'un nouveau Christ ou tentative d'adhésion à une église existante).

8 J'ai mieux compris la nature de ces enjeux lorsque j'ai étudié au Togo « en direct " le fonctionnement des polythéismes locaux. J'avais en effet sous les yeux au Togo, dans les années 1970 et 1980, une organisation religieuse qui évoquait ce que l'on me racontait dans le sud de la Côte-d'Ivoire des cultes officiellement disparus.

9 Les dieux des panthéons que l'on trouve au Nigeria, au Bénin ou au Togo rappellent à bien des égards les dieux grecs tels que les a analysés Jean-Pierre Vernant. Ce sont incontestablement des personnages, dont on raconte les aventures dans les mythes. Mais, pour le reste, ce sont davantage des « puissances » que des « personnes ». Ils sont additionnables les uns aux autres, comme des éléments chimiques, à la fois singuliers et pluriels. Ces recompositions aboutissent parfois à la création d'entités bisexuées; parfois aussi cette ambivalence sexuelle traverse chaque personnage singulier. Au total, ce sont des forces qu'il faut savoir maitriser et contrôler pour agir sur d'autres hommes ou s'en protéger. Car le «dieu», le "vodun» est avant tout un médiateur entre les hommes, mais un médiateur très particulier, un moyen d'action, une arme ou un bouclier. La force du dieu dans sa manifestation locale, c'est aussi celle de son prêtre ou de son initié, appelé à mettre en œuvre cette force.

10 Car le polythéisme, ce n'est pas seulement la pluralité des personnages divins, c'est plus encore la pluralité de chacun de ces personnages. Un dieu - par exemple Hevieso, dieu du tonnerre qui par nombre de ses attributs évoque assez précisément Zeus - est un personnage connu, dont on décrit le caractère et à propos duquel on raconte certaines anecdotes. Mais il est présent à des milliers d'exemplaires dans tous les villages, dans 
les demeures des uns et des autres, et l'on entend des appréciations du type: "Cet Hevieso est particulièrement fort" ou "plus ou moins fort que tel ou tel autre Hevieso ». Le nom Hevieso, comme tous ses homologues des panthéons yorouba ou ewe, désigne à la fois un personnage de mythe, dont on connaît les attributs et les fonctions, et une multitude de dispositifs singuliers, comparables, parce qu'ils sont sujets aux mêmes prescriptions et aux mêmes interdits, mais tous différents, inégalement puissants et efficaces, irrémédiablement singuliers et locaux.

11 Une des conséquences de cet état de choses, c'est que le sacré comme séparation du profane n'est guère repérable dans l'espace : il est partout. Les demeures sont hérissées de défenses mystiques très visibles; à l'intérieur des demeures, des autels sont consacrés à des divinités qui au fil du temps, à l'occasion de divers événements, ont été associées à la vie des familles, des lignages. Cela n'empêche d'ailleurs pas que telle ou telle de ces divinités fasse aussi l'objet d'un culte plus collectif au niveau du village ou de la région. Il y a eu dans cette partie de l'Afrique des royaumes et des vodun associés à la maison royale. Mais au total, c'est le décentrement de la vie religieuse qui frappe et la projection dans le temps de la coupure sacré/profane (il y a le temps sacré du culte, des initiations et des diverses célébrations dans les mêmes lieux où se déroulent les activités de la vie profane). Ce décentrement tient à la nature même de ce que l'on appelle les dieux du polythéisme, qui sont avant tout conçus comme des instruments de relation entre les hommes.

Puissances plus que personnes, les vodun servent essentiellement à l'interprétation des événements (notamment la maladie et la mort) et à la gestion des relations avec les autres individus sous des modalités agressives ou défensives. Leur présence se manifeste aussi bien dans l'espace public que sur ou même dans le corps des individus. Le vodun Legba illustre particulièrement bien cette plasticité. Comme l'Hermès grec, Legba est le dieu des carrefours, des marchés et des marchands, des limites et des frontières. On trouve des figurations de Legba au croisement des chemins, sur la place des villages, mais aussi à l'entrée de chaque demeure en plusieurs exemplaires. Car chacun a son Legba et même deux Legba : un à l'entrée de la demeure, pour se protéger des attaques d'autrui, l'autre à côté de l'endroit où l'on dort, pour se protéger de ses propres pulsions agressives. Legba est étroitement associé à d'autres entités ( $\mathrm{Fa}$, Kpoli) qui sont conçues à la fois comme des vodun et comme des éléments de la personne individuelle, les unes héritées et transmissibles, les autres périssables et fugitives. Cette impressionnante dimension symbolique a valu à Legba une carrière de voyageur (on le retrouve en Haïti et en Amérique du Sud) et aussi une réputation sulfureuse (on en a fait parfois un équivalent du diable).

Les systèmes polythéistes africains obéissent à une logique de l'immanence. Identité et altérité y sont étroitement liées; il existe une certaine porosité entre les individus pris dans le jeu des relations et des influences; les vivants ne s'y opposent pas plus aux morts que les hommes aux dieux: chacune de ces catégories n'est qu'une transformation des autres. Il est souvent dit explicitement que les vodun sont d'anciens hommes, des ancêtres donc, qui reviennent dans les phénomènes de possession. Cette conception circulaire vaut pour tous et pour chacun. D'où ce que j'ai appelé, faute d'un meilleur terme, le décentrement du culte.

De ce point de vue, le monothéisme s'oppose au polythéisme sur plusieurs plans. Dire que chaque homme a une relation directe à Dieu, c'est l'individualiser, et l'action des prophètes africains a parfois été interprétée comme un effort pour libérer l'individu 
des conceptions "persécutives", dans le langage des psychologues, qui faisaient dépendre son existence et même sa définition de ses relations avec autrui. Cette individualisation, dans la perspective monothéiste, s'accompagne logiquement d'une conception de la communauté des individus ainsi libérés. Au sein de cette communauté, Dieu n'est pas le médiateur des relations entre les hommes, comme dans les systèmes polythéistes (même si le culte des saints joue parfois un rôle analogue), mais ces relations sont en principe l'expression de la relation à Dieu: il y a des manières chrétiennes de se comporter avec autrui.

Cette communauté, pour exister, doit se voir, se réunir et se reconnaître. Le lien singulier à Dieu et le lien de solidarité entre les croyants : c'est cela que symbolise et met en espace l'Église comme construction matérielle. Les prophètes africains auprès desquels je travaillais dans les années 1970 avaient l'intuition de cette nécessité et leurs approximations en ce domaine ne doivent pas être comprises comme une simple péripétie coloniale et postcoloniale. Quelques Églises africaines ont vu le jour au Congo et en Côte-d'Ivoire et survivent; mais aujourd'hui la pénétration des évangélistes est impressionnante en Afrique comme en Amérique latine, où l'Église catholique résiste en amplifiant les moyens traditionnels d'occupation de l'espace public, par exemple en organisant les processions de la semaine sainte et en ressuscitant les fastes de la liturgie. Ce n'est pas pour rien, non plus, que les musulmans, en Europe, font pression pour édifier des mosquées. Sacralité monothéiste et architecture ont partie liée. Les grands prêtres de la société de consommation le savent aussi, qui élèvent des tours gigantesques aux quatre coins de la planète et inscrivent dans le ciel le nom de leurs entreprises.

\section{AUTEUR}

\section{MARC AUGÉ}

École des hautes études en sciences sociales, Paris. 